European

Thyroid Journal
Eur Thyroid J 2014;3:227-233

DOI: $10.1159 / 000366274$
Received: June 10, 2014

Accepted after revision: August 4, 2014

Published online: October 15, 2014

\title{
Novel NKX2-1 Frameshift Mutations in Patients with Atypical Phenotypes of the Brain-Lung-Thyroid Syndrome
}

\author{
Tiziana de Filippis ${ }^{a, b}$ Federica Marelli ${ }^{a}, b$ Maria Cristina Vigone ${ }^{c}$ \\ Marianna Di Frenna ${ }^{c}$ Giovanna Weber ${ }^{c}$ Luca Persani $^{a}$, b, d \\ a Laboratory of Endocrine and Metabolic Research, and b Division of Endocrinology and Metabolic Diseases, IRCCS \\ Istituto Auxologico Italiano, ' Department of Pediatrics, Vita-Salute University, San Raffaele Scientific Institute, and \\ ${ }^{\mathrm{d}}$ Department of Clinical Sciences and Community Health, University of Milan, Milan, Italy
}

\section{Key Words}

NKX2-1 · Congenital hypothyroidism · Brain-lung-thyroid syndrome Choreoathetosis · Thyroid dysgenesis .

Thyroid ectopy

\begin{abstract}
Objectives: To verify the involvement of NKX2-1 gene in infants with brain-lung-thyroid (BLT) syndrome and hypothyroid phenotypes variable among congenital hypothyroidism $(\mathrm{CH})$ or idiopathic mild hypothyroidism (IMH) of postnatal onset. Methods: The candidates were selected by a case-finding approach in $130 \mathrm{CH}$ and $53 \mathrm{IMH}$ infants. The NKX2-1 gene was analyzed by direct sequencing and multiplex ligation-dependent probe amplification. The variants were studied in vitro, by expression analyses and luciferase bioassay. Results: Four cases ( $3 \mathrm{CH}$ and $1 \mathrm{IMH}$ ) consistent with BLT syndrome were identified. Two children were affected with respiratory distress and $\mathrm{CH}$, but wild-type NKX21 gene. The remaining two presented choreic movements and no pulmonary involvement, but discrepant thyroid phenotypes: one had severe $\mathrm{CH}$ with lingual ectopy and the other one IMH with gland in situ. They were carriers of new de novo heterozygous frameshift mutations of NKX2-1 (c.177delG and c.153_166del14). The c.177delG leads to a
\end{abstract}

prematurely truncated protein (p.H60Tfs X11) with undetectable activity in vitro. The c.153_166del14 leads to the generation of an elongated aberrant protein (p.A52Rfs X351) able to translocate into the nucleus, but completely inactive on a responsive promoter. Conclusions: Two novel heterozygous frameshift mutations of NKX2-1 were identified in 2 cases selected on the basis of a BLT-like phenotype among 183 hypothyroid infants. The atypical hypothyroid phenotypes of these 2 children ( $\mathrm{CH}$ with lingual ectopy or IMH of postnatal onset) further expand the clinical spectrum that can be associated with NKX2-1 mutations.

(c) 2014 European Thyroid Association Published by S. Karger AG, Basel

\section{Introduction}

In humans, heterozygous loss-of-function mutations in NKX2-1 gene (OMIM \#600635) have been reported to cause a complex phenotype called brain-lung-thyroid (BLT) syndrome (OMIM \#610978) [1-7]. The BLT syndrome is a rare disease characterized by a highly variable penetrance and expressivity and combining neurological manifestations (hypotonia evolving into benign chorea and ataxia), pulmonary disease (neonatal respiratory dis-

\begin{tabular}{ll}
\hline KARGER & @ 2014 European Thyroid Association \\
& $\begin{array}{l}\text { Published by S. Karger AG, Basel } \\
\text { 2235-0640/14/0034-0227\$39.50/0 }\end{array}$ \\
$\begin{array}{l}\text { E-Mail karger@karger.com } \\
\text { www.karger.com/etj }\end{array}$ &
\end{tabular}

Luca Persani, $\mathrm{MD}, \mathrm{PhD}$

Department of Clinical Sciences and Community Health, University of Milan San Luca Hospital, IRCCS Istituto Auxologico Italiano

Piazzale Brescia 20, IT-20149 Milan (Italy)

E-Mail luca.persani@unimi.it 
tress and/or interstitial lung disease), and congenital hypothyroidism $(\mathrm{CH})$ of variable severity, associated either with athyreosis, hypoplasia or an apparently normal gland in situ [1-7], but not with ectopy as recently indicated also in the document of the $\mathrm{CH}$ Consensus Conference Group [8]. NKX2-1 variants had also been reported in isolated benign hereditary chorea (BHC; OMIM \#118700) [9]. Here, we examined the frequency of a possible BLT involvement among 183 children followed for hypothyroidism of congenital or early postnatal onset and identified two novel heterozygous NKX2-1 frameshift mutations that were associated with typical neurological manifestations, absent pulmonary involvement and atypical thyroid phenotypes, such as lingual ectopy or non-autoimmune mild hypothyroidism.

\section{Materials and Methods}

\section{Patients}

Among 183 children followed at our center for hypothyroidism ( $\mathrm{CH}$ in 130 or early postnatal onset between 1 and 12 months of age in 53), we selected the patients who were candidates for NKX21 analysis on the basis of clinical manifestations consistent with a BLT syndrome. The local ethics committee approved the study and informed consent for genetic analyses was obtained from the parents of all children.

\section{Genetic Analysis}

Genomic DNA was extracted from peripheral blood lymphocytes using the GeneCatcher gDNA $96 \times 10 \mathrm{ml}$ Automated Blood Kit (Life Technologies, Foster City, Calif., USA). The entire coding regions and splicing sites of NKX2-1,PAX8 and FOXE1 genes were screened by direct sequencing using the Big Dye Terminator Kit (Life Technologies) and analyzed on the ABI Prism 3100 automated sequencer (Life Technologies). Primer sequences are available upon request. Possible deletions or alterations in the $5^{\prime}$ region of NKX2-1 gene were also analyzed by Multiplex Ligation Probe Amplification (SALSA MLPA P319 Thyroid Probemix; MRCHolland, Amsterdam, The Netherlands) or direct sequencing, respectively.

\section{Mutagenesis}

The plasmid pSG5_hNKX2-1_WT was kindly provided by Prof. Refetoff [2]. The NKX2-1 variants (p.A52R $f$ X 351 and p.H60T $f$ X 11 ) were obtained by mutagenesis using the GeneArt ${ }^{\circledR}$ Site-Directed Mutagenesis PLUS System (Life Technologies). Each variant was confirmed by direct sequencing.

\section{Transient Transfections}

NIH-3T3 cells were grown in Dulbecco's modified Eagle medium (D-MEM) (1×), with GlutaMAX ${ }^{\mathrm{TM}}$ I supplemented with $10 \%$ new calf serum, and penicillin 1\%-streptomycin 1\% (all from Life Technologies). All transient transfections were performed using Lipofectamine ${ }^{\mathrm{TM}} 2000$ Transfection Reagent (Life Technologies) in NIH-3T3 cells (80-90\% confluence). Depending on the type of experiment, variable amounts of plasmids were mixed with Lipo- fectamine $^{\mathrm{TM}} 2000$ in serum-free OptiMEM medium (Life Technologies) for $20 \mathrm{~min}$ before being added to the cells. After $4-5 \mathrm{~h}$ of incubation, the transfection medium was removed and complete medium was added for another $24 \mathrm{~h}$.

\section{Expression Studies}

Total RNA was extracted from transfected cells and the expression levels of the different constructs were evaluated by real-time quantitative PCR (RT-QPCR), as previously described [10]. The primers are available upon request.

For protein analyses, cells were lysed in $300 \mu \mathrm{l}$ of lysis buffer ( 20 mM Tris pH 8, $150 \mathrm{~mm} \mathrm{NaCl}, 1 \%$ Triton X-100, 5 mM EDTA, 0.2\% BSA) containing protease inhibitors (Complete; Roche, Indianapolis, Ind., USA). The lysates were loaded at two different concentrations (5 and $20 \mu \mathrm{g}$ ) on NuPAGE 4-12\% Bis-Tris gel (Life Technologies) and electrophoretically transferred to nitrocellulose membrane (GE Healthcare, Little Chalfont, UK). Membranes were probed overnight at $4{ }^{\circ} \mathrm{C}$ with two different anti-NKX2-1 monoclonal antibodies (Clone 8G7G3/1; Dako A/S, Glostrup, Denmark, or clone SPT24; Monosan, Uden, The Netherlands) directed against the N-terminal domain or with anti-tubulin (Thermo Scientific Pierce Antibodies, Rockford, Ill., USA), and then incubated for $1 \mathrm{~h}$ at room temperature with secondary antibody. Detection was performed utilizing the ECL Plus Western Blotting Detection Reagents (GE Healthcare).

For the immunofluorescence experiments, cells were plated on glass coverslips and $24 \mathrm{~h}$ after transfections they were fixed in $4 \%$ paraformaldehyde, and incubated for $1 \mathrm{~h}$ with the monoclonal antibody (Clone 8G7G3/1; Dako). The Alexa Fluor 488 goat antimouse IgG (Life Technologies) was used for detection, and signal was visualized using a confocal microscope Nikon C2+.

\section{Luciferase Reporter Assay}

NIH-3T3 cells were transiently transfected with a mixture of $500 \mathrm{ng}$ of the firefly luciferase construct driven by the human thyroglobulin enhancer/promoter [2], $20 \mathrm{ng}$ of the pRL-TK Renilla construct (Promega, Madison, Wisc., USA), as an internal control for transfection efficiency, and variable amounts of the WT or mutant NKX2-1 constructs. The cells were harvested at $24 \mathrm{~h}$, and firefly/Renilla luciferase activities were measured using the Dual-Luciferase Reporter Assay System (Promega). The efficiency of transfection was normalized to the activity of Renilla construct, and the activity was expressed as ratio of firefly to Renilla Luciferase activities. Three independent experiments were performed in triplicate.

\section{Statistical Analysis}

Statistical analyses were carried out using PRISM 4.0 software and we performed a one-way ANOVA analysis followed by the Kruskal-Wallis comparison test.

\section{Results}

\section{Patients}

Only 4 out 183 hypothyroid children were candidates for NKX2-1 analysis due to clinical manifestations consistent with BLT syndrome. Cases 1 and 2 were born at term, they were affected with $\mathrm{CH}$ due to thyroid hypoplasia 
Table 1. Data of patients with NKX2-1 mutations

a Clinical and biochemical data

\begin{tabular}{lll}
\hline & Patient 3 & Patient 4 \\
\hline Neonatal TSH screening on whole blood (normal values $<10 \mathrm{mU} / \mathrm{l})$ & $>100$ & negative \\
Age at diagnosis of hypothyroidism & 9 days & 10 months \\
TSH at diagnosis (normal values $0.4-6.3 \mathrm{mU} / \mathrm{l})$ & $>200$ & 15.9 \\
FT4 at diagnosis (normal values $1.78-4.2 \mathrm{ng} / \mathrm{dl})$ & 0.2 & 1.4 \\
Thyroid US scan & ectopy & normal \\
${ }^{99}$ Tc thyroid scintigraphy & ectopy & - \\
Respiratory disease & none & none \\
Age at onset of choreoathetosis & 6 months & 10 months \\
Brain MRI & normal & normal \\
NKX2-1 mutation & p.H60T $f$ XX1 & p.A52R $f$ X351 \\
\hline
\end{tabular}

b Neuropsychological outcome

\begin{tabular}{llrrr}
\hline & \multicolumn{2}{c}{ Patient 3 } & \multicolumn{2}{c}{$\begin{array}{c}\text { Patient } 4 \\
(2 \text { years })\end{array}$} \\
\cline { 2 - 4 } & 6 months & 1 year & 4 years & \\
\hline Developmental quotient & 110 & 87 & 89 & 75 \\
Locomotor quotient & 107 & 61 & 56 & 60 \\
Personal-social quotient & 110 & 81 & 109 & 90 \\
Hearing and language quotient & 121 & 96 & 113 & 60 \\
Eye and hand coordination quotient & 103 & 95 & 65 & 72 \\
Performance quotient & 108 & 104 & 87 & 90 \\
\hline
\end{tabular}

Patient 3 underwent repeated neuropsychological evaluations at 6 months, 1 year and 4 years of age showing a progressive deterioration of most quotients, particularly evident in the locomotor and eye-hand coordination quotients consistent with the type of neurological defect.

(neonatal TSH screening $>100 \mathrm{mU} / \mathrm{l}$ ), and respiratory distress syndrome since the first weeks after birth. Both died of respiratory distress syndrome but were negative for NKX2-1 alterations, also after the analysis of the regulatory regions of the gene or the search of possible deletions.

The remaining 2 children had no lung disease, but neurological defects despite the early start of L-thyroxine treatment in 1 case and the normal results of TSH screening at birth in the other. Patient 3 was born after an uneventful pregnancy and delivery at term. He was suspected to have $\mathrm{CH}$ at neonatal screening (table 1 ). Serum evaluation confirmed severe hypothyroidism with high TSH (>200 mU/l) and low $\mathrm{FT}_{4}$ serum levels $(0.2 \mathrm{ng} / \mathrm{dl}$, normal range for age $1.78-4.2 \mathrm{ng} / \mathrm{dl})$ [11]. Thyroid ${ }^{99} \mathrm{Tc}$ scintigraphy and US scan revealed a lingual thyroid ectopy, therefore $\mathrm{L}_{-} \mathrm{T}_{4}$ replacement therapy was introduced on the 9 th day of postnatal life at $12.2 \mu \mathrm{g} / \mathrm{kg} /$ day. No respiratory problems occurred in the neonatal period or over the first years of life, but at 6 months of age the child mani-
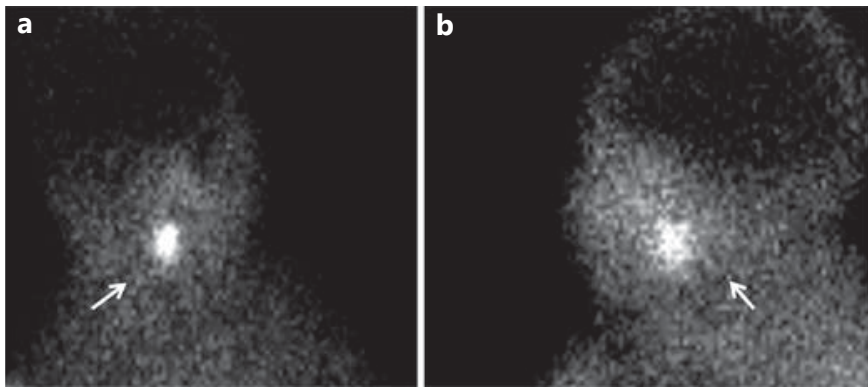

Fig. 1. a, b Scintiscan showing the lingual thyroid ectopy (arrows) in patient 3 with the p.H60TfsX11 mutation.

fested psychomotor retardation with athetosic movements and spasticity. Brain MRI as well as tests for metabolic disorders were negative (fig. 1a, b).

Patient 4 was born at term after an uneventful pregnancy. He had a negative $\mathrm{CH}$ screening (blood TSH at 


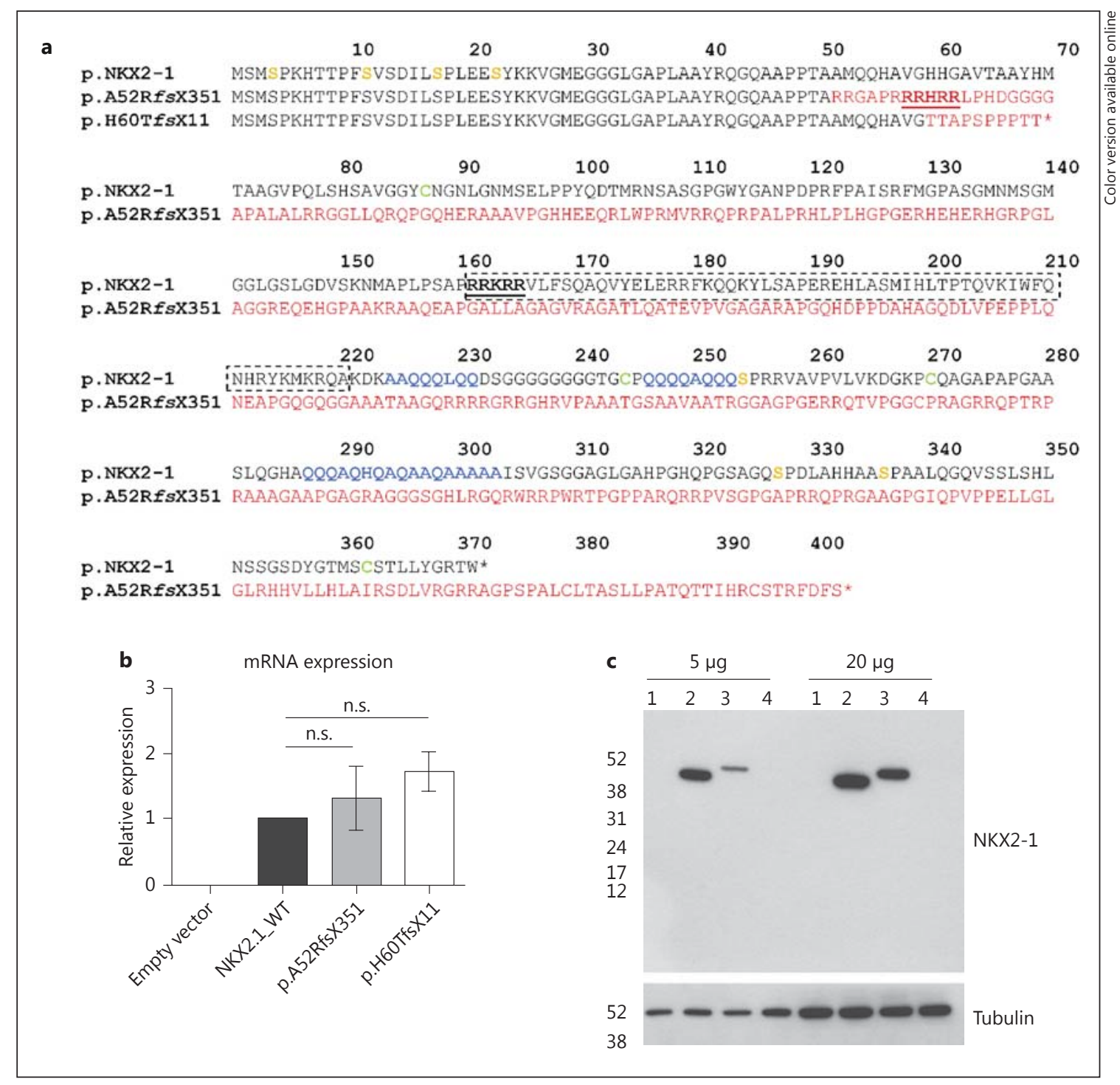

Fig. 2. a Deduced amino acid sequences of mutant proteins (divergent sequences in red; colors refer to the online version only) compared with the NKX2-1 WT protein (in black). The canonical and putative NLS signals of NKX2-1 are in bold and underlined. The described homeodomain is included in a dotted box, the glutamine-rich regions are highlighted in blue, and the Cys involved in dimerization are in green, and the Ser involved in phosphorylation are in yellow [12-14]. b, c mRNA and protein expression. NIH3T3 cells were transiently transfected with the different constructs: $1=$ empty vector; $2=$ NKX2- $1 \mathrm{WT} ; 3=$ p.A52RfsX351, and $4=$ p.H60T $f$ X11. The mRNA and proteins expression levels were an-

birth $<10 \mathrm{mU} / \mathrm{l}$ ) [11]. At 6 months, postural defects were noted in association with failure to thrive; at 10 months of age the baby came to our attention because of the finding of idiopathic mild hypothyroidism (IMH; serum TSH values $14.5-15.9 \mathrm{mU} / \mathrm{l}$, normal values $0.4-6.3$ ) with nor- alyzed by RT-QPCR (b) and Western blot (c), respectively. In RTQPCR, the mRNA levels were not statistically different (one-way ANOVA followed by Kruskal-Wallis comparison test; n.s. = not significant). At Western blot, similar results were obtained with two different anti-NKX2-1 antibodies directed against the N-terminal domain of NKX2-1. Note the 40-kDa size of NKX2-1 WT and the increased weight of the elongated mutant p.A52R $f$ X 351 . The protein expression of the p.H60T $f s \mathrm{X} 11$ was not detectable despite the conservation of the first 60 residues, and even after the loading of $20-\mu \mathrm{g}$ proteins.

mal $\mathrm{FT}_{4}$ levels (normal range for age 1.5-2.4 ng/dl) (table 1). Thyroid ultrasound showed a normal gland, the antithyroid antibodies were negative and $\mathrm{L}_{-} \mathrm{T}_{4}$ replacement therapy was started. The neurological evaluation revealed hypotonia and choreoathetosis. At 2 years of age 
Fig. 3. a Subcellular localization of NKX2-1 WT and p.A52RfsX351 proteins. Nuclei are stained in blue with DAPI, and green fluorescence stains the NKX2-1 variants; both of them are efficiently targeted to the nucleus (colors refer to the online version only). b A reporter luciferase expression vector was co-transfected into NIH-3T3 cells with the NKX2-1 WT or mutated plasmids. The cDNA amounts and combinations used in transfection experiments are indicated. Promoter activity is expressed as fold induction relative to the activity of the empty vector. Luciferase activity is normalized to Renilla activity derived from the cotransfection of pRL-TK vector. ${ }^{*} \mathrm{p}<0.0001$ vs. 250 ng WT-NKX2-1; ${ }^{* *} \mathrm{p}$ not significant vs. 125 ng WT-NKX2-1 + 125 ng empty vector.

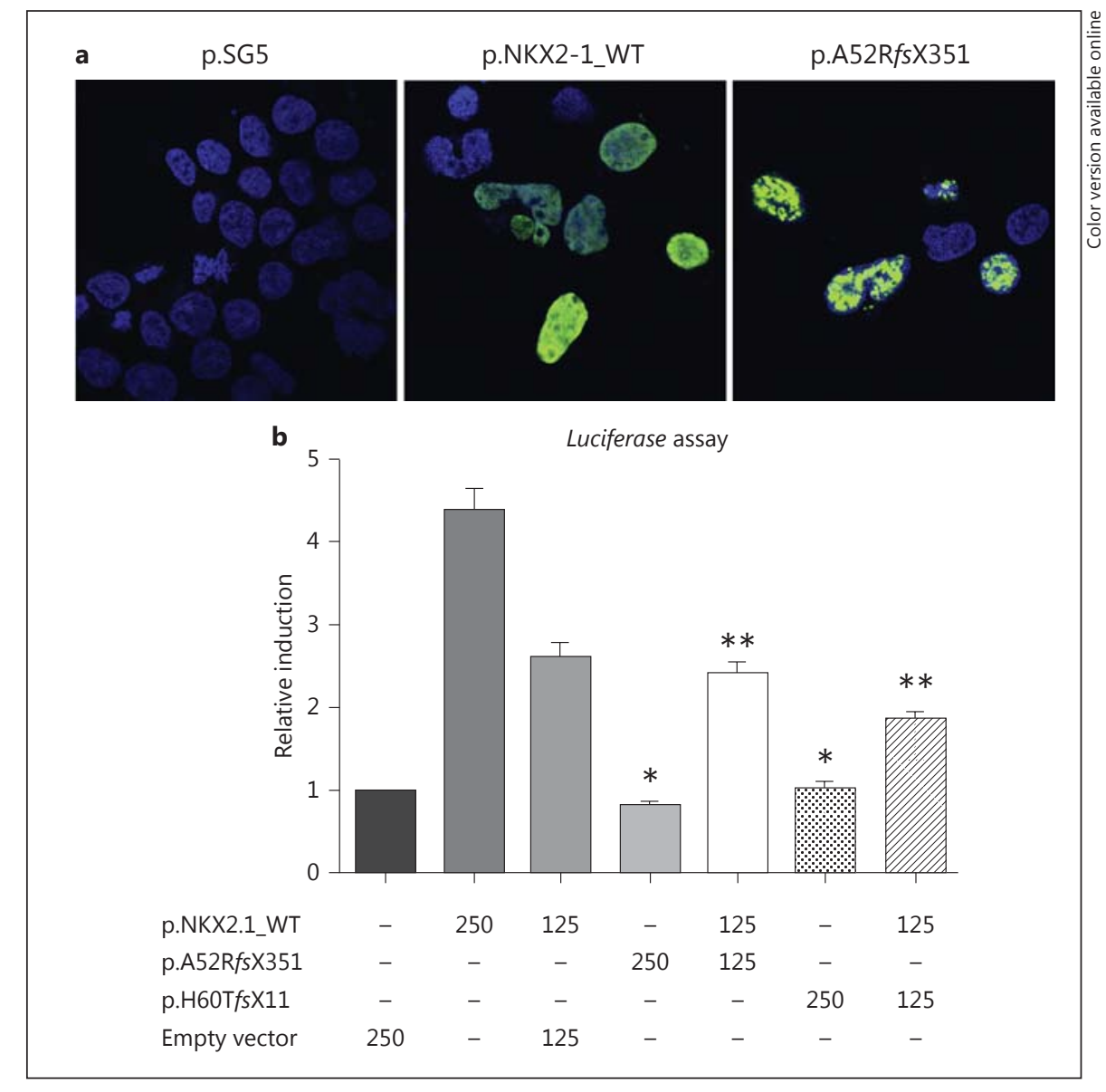

neurological defects persisted and speech was modestly retarded, therefore we performed brain MRI that revealed normal myelinization. No respiratory defect occurred during the first years of life.

The neuropsychological outcome was poor in both cases, and a progressive deterioration was seen in case 3 over the years (table 1).

\section{Genetic Analysis}

Sequencing analysis of the NKX2-1 gene identified two distinct new heterozygous mutations. Both mutations are located in exon 2 [12]. In case 3, we detected a deletion at position 177 (c.177 $\Delta \mathrm{G}$, reference sequence NM_003317.3) leading to a frameshift affecting the histidine- 60 and generating a premature stop codon (p.H60TfsX11) (fig. 2a). In case 4 , we found the deletion of 14 nucleotides at position 153 (c.153_166 14, reference sequence NM_003317.3) leading to a frameshift involving also exon 3 and the $3^{\prime}$ untranslated region, and generating a postponed stop codon (p.A52RfsX351) (fig. 2a). The aberrant protein is predicted to be 31 amino acids longer than wild-type NKX2-1 pro- tein. Due to premature truncation or aberrant divergent sequence of the elongated protein, both mutants lack the described homeodomain, glutamine-rich domains and the Cys involved in dimerization of NKX2-1 protein [12-14].

Both mutations occurred de novo in the 2 probands. Both patients have wild-type PAX8- and FOXE1-coding sequences.

\section{Expression and Subcellular Localization of the NKX2-1 Mutants}

Despite the detection of both variant transcripts at RT-QPCR experiments (fig. 2b), the expression of the only mutant protein p.A52RfsX351 can be detected in transfected cells by Western blot (fig. 2c). At confocal microscopy (fig. 3a), both WT and p.A52RfsX351 NKX2-1 proteins are efficiently targeted into the nucleus, despite the loss of the canonical nuclear localization signal (NLS) at amino acid residues 161-165 (RRKRR). The analysis of the mutated sequence revealed the generation of a potential alternative NLS at position 58-62 (RRHRR) (fig. 2a). 


\section{Luciferase Assay}

The luciferase activity detected in cells transiently transfected with 250 ng of WT-NKX2-1 cDNA is about fourfold higher over baseline, whereas no activation of the TG promoter can be detected with both mutant proteins (fig. 3b). In cells co-transfected with equal amounts of WT and mutant NKX2-1, thus mimicking the heterozygous condition in vivo, we detect about $50 \%$ of the WT activity (fig. $3 \mathrm{~b}$ ). The co-transfection of different $\mathrm{WT} /$ mutant proportions fails to show interference by mutant proteins on WT bioactivity.

\section{Discussion}

Among a series of 183 infants followed for hypothyroidism, the BLT phenotypes suggesting a possible NKX21 involvement were detected in 4 cases. However, we identified two new heterozygous frameshift mutations in NKX2-1 gene in only 2 of them, showing typical neurological defects but a completely different thyroid phenotype. One was affected with severe $\mathrm{CH}$, whereas the second was negative at neonatal TSH screening, but was diagnosed with IMH at the age of 10 months during examinations for the onset of choreoathetosis. These thyroid phenotypes are atypical in patients with NKX2-1 pathogenic variants [4-8] and patient 3 represents the first $\mathrm{CH}$ case of lingual ectopy associated with a mutation in this gene [8]. Differently, the finding of mild hypothyroidism of postnatal onset in the second child underscores the importance to evaluate thyroid function in patients coming to the pediatrician or neurologist attention for choreic movements. Indeed, NKX2-1 mutations were previously reported in patients with isolated $\mathrm{BHC}$ [9].

The pathogenic role of both frameshift variations is indeed supported by several findings: first, the concomitant de novo appearance of the genetic and phenotypic defects in the two families [15]; in addition, in vitro experiments demonstrate the complete loss-of-function for both mutations. The p.H60 $f f$ X 11 truncated protein was undetectable at Western blot, whereas the aberrant elongated protein p.A52R $f$ X 351 is translated and can translocate into the nucleus of transfected cells, but fails to activate the $T G$ promoter and to interfere with WT-NKX2-1 activity. Indeed, the sequences of NKX2-1 functional domains are lacking in p.H60TfsX11 or are completely divergent in p. A52RfsX351 (fig. 2), consistent with the lack of interference on wild-type NKX2-1 activity. A bioinformatic analysis revealed that, despite causing the loss of the canonical NLS (RRKRR) [7], the frameshift mutation p.A52R $f$ X351 generates a potential novel NLS sequence (RRHRR), which could justify the nuclear localization. This prediction is supported by the similar chemical and physical properties of Lys $(\mathrm{K})$ and His $(\mathrm{H})$ that belong to the same group of basic amino acids at neutral $\mathrm{pH}$, together with $\operatorname{Arg}(\mathrm{R})$.

Despite their complete loss-of-function, the frameshift mutations are associated with highly discrepant phenotypes, indicating the existence of modifying genes or factors that contribute to the variable expression of BLT phenotype. In agreement with this hypothesis, experimental studies showed the possible existence of multigenic defects underlying the pathogenesis of $\mathrm{CH}$ and thyroid dysgenesis [16-19].

\section{Conclusions}

We identified novel heterozygous NKX2-1 frameshift mutations in 2 patients with atypical phenotypes of BLT syndrome: choreoathetosis associated with discrepant thyroid phenotypes (severe $\mathrm{CH}$ with lingual ectopy or mild hypothyroidism of early postnatal onset). Interestingly, the NKX2-1 mutation p.H60TfsX11 is the first genetic abnormality that can be linked with thyroid ectopy. Therefore, the spectrum of clinical expressivity of heterozygous NKX2-1 mutations is wider than previously thought.

\section{Acknowledgements}

We thank all members of the two affected families for their collaborative participation in this study. We are grateful to Prof. S. Refetoff and Dr. A. Dumitrescu for providing the p.hNKX2-1 WT plasmid and reporter luciferase expression vector. This work was partially supported by the Ricerca Finalizzata program of the Italian Ministry of Health (grant RF-2010-2309484).

\section{Disclosure Statement}

The authors have no conflicts of interest to disclose.

References

1 Krude H, Schütz B, Biebermann H, von Moers A, Schnabel D, Neitzel H, Tönnies $H$, Weise D, Lafferty A, Schwarz S, DeFelice M, von Deimling A, van Landeghem F, DiLauro R, Grüters A: Choreoathetosis, hypothyroidism, and pulmonary alterations due to human NKX2-1 haploinsufficiency. J Clin Invest 2002;109:475-480.

2 Pohlenz J, Dumitrescu A, Zundel D, Martiné U, Schönberger W, Koo E, Weiss RE, Cohen RN, Kimura S, Refetoff S: Partial deficiency of thyroid transcription factor 1 produces predominantly neurological defects in humans and mice. J Clin Invest 2002;109:469-473. 
3 Guillot L, Carré A, Szinnai G, Castanet M, Tron E, Jaubert F, Broutin I, Counil F, Feldmann D, Clement A, Polak M, Epaud R: NKX2-1 mutations leading to surfactant protein promoter dysregulation cause interstitial lung disease in 'brain-lung-thyroid syndrome'. Hum Mutat 2010;31:E1146-E1162.

-4 Thorwarth A, Schnittert-HübenerS, Schrumpf P, Müller I, Jyrch S, Dame C, Biebermann H, Kleinau G, Katchanov J, Schuelke M, Ebert G, Steininger A, Bönnemann C, Brockmann K, Christen HJ, Crock P, deZegher F, Griese M, Hewitt J, Ivarsson S, Hübner C, Kapelari K, Plecko B, Rating D, Stoeva I, Ropers HH, Grüters A, Ullmann R, Krude H: Comprehensive genotyping and clinical characterisation reveal 27 novel NKX2-1 mutations and expand the phenotypic spectrum. J Med Genet 2014;51:375-387.

5 Carrè A, Szinnai G, Castanet M Sura-Trueba S, Tron E, Broutin-L'Hermite I, Barat P, Goizet C, Lacombe D, Moutard ML, Raybaud C, Raynaud-Ravni C, Romana S, Ythier H, Léger J, Polak M: Five new TTF1/NKX2-1 mutations in brain-lung-thyroid syndrome: rescue by PAX8 synergism in one case. Hum Mol Genet 2009; 18:2266-2276.

6 Montanelli L, Tonacchera M: Genetics and phenomics of hypothyroidism and thyroid dys- and agenesis due to PAX8 and TTF1 mutations. Mol Cell Endocrinol 2010;322: 64-71.

7 Nettore IC, Mirra P, Ferrara A Sibilio A, Pagliara V, Kay CS, Lorenzoni PJ, Werneck LC, Bruck I, Dos Santos LH, Beguinot F, Salvatore D, Ungaro P, Fenzi G, Scola RH, Macchia PE: Identification and functional characterization of a novel mutation in the NKX2-1 gene: comparison with the data in the literature. Thyroid 2013;23:675-682.
8 Léger J, Olivieri A, Donaldson M, Torresani T, Krude H, van Vliet G, Polak M, Butler G; ESPE-PES-SLEP-JSPE-APEG-APPESISPAE; Congenital Hypothyroidism Consensus Conference Group: European Society for Paediatric Endocrinology consensus guidelines on screening, diagnosis, and management of congenital hypothyroidism. J Clin Endocrinol Metab 2014;99:363-384.

-9 Breedveld GJ, van Dongen JW, Danesino C, Guala A, Percy AK, Dure LS, Harper P, Lazarou LP, van der Linde $\mathrm{H}$, Joosse $\mathrm{M}$, Gruters A, MacDonald ME, de Vries BB, Arts WF, Oostra BA, Krude H, Heutinl P: Mutations in TITF-1 are associated with benign hereditary chorea. Hum Mol Genet 2002;11:971-979.

10 Calebiro D, de Filippis T, Lucchi S, Martinez F, Porazzi P, Trivellato R, Locati M, Beck-Pec$\operatorname{coz}$ P, Persani L: Selective modulation of protein kinase A I and II reveals distinct roles in thyroid cell gene expression and growth. Mol Endocrinol 2006;20:3196-3211.

-11 Corbetta C, Weber G, Cortinovis F, Calebiro D, Passoni A, Vigone MC, Beck-Peccoz P, Chiumello G, Persani L: A 7-year experience with low blood TSH cutoff levels for neonatal screening reveals an unsuspected frequency of congenital hypothyroidism. Clin Endocrinol (Oxf) 2009;71:739-745.

12 Guazzi S, Price M, De Felice M, Damante G, Mattei MG, Di Lauro R: Thyroid nuclear factor 1 contains a homeodomain and displays a novel DNA binding specificity. EMBO J 1990; 9:3631-3639.
13 De Felice M, Damante G, Zannini M, FrancisLang H, Di Lauro R: Redundant domains contribute to the transcriptional activity of the thyroid transcription factor 1. J Biol Chem 1995;270:26649-26656.

14 Moya CM, Perez de Nanclares G, Castaño L, Potau N, Bilbao JR, Carrascosa A, Bargadá M, Coya R, Martul P, Vicens-Calvet E, Santisteban P: Functional study of a novel single deletion in the TITF1/NKX2.1 homeobox gene that produces congenital hypothyroidism and benign chorea but not pulmonary distress. J Clin Endocrinol Metab 2006;91:18321841.

15 Tavtigian SV, Greenblatt MS, Goldgar DE, Boffetta P: Assessing pathogenicity: overview of results from the IARC Unclassified Genetic Variants Working Group. Hum Mutat 2008;29:1261-1264.

16 Amendola E, Sanges R, Galvan A, Dathan N, Manenti G, Ferrandino G, Alvino FM, Di Palma T, Scarfò M, Zannini M, Dragani TA, De Felice M, Di Lauro R: A locus on mouse chromosome 2 is involved in susceptibility to congenital hypothyroidism and contains an essential gene expressed in thyroid. Endocrinology 2010;151:1948-1958.

17 Fagman H, Nilsson M: Morphogenesis of the thyroid gland. Mol Cell Endocrinol 2011;323: 35-54.

18 De Felice M, Di Lauro R: Minireview: intrinsic and extrinsic factors in thyroid gland development: an update. Endocrinology 2011; 152:2948-2956.

19 Nettore IC, Cacace V, De Fusco C, Colao A, Macchia PE: The molecular causes of thyroid dysgenesis: a systematic review. J Endocrinol Invest 2013;36:654-664. 